\title{
O efeito da orientação preventiva multiprofissional em pacientes com diabetes mellitus
}

\author{
The effect of multiprofessional preventive guidance in diabetes mellitus patients
}

\author{
El efecto de la orientación preventiva multiprofesional en pacientes con diabetes mellitus
}

Daniel Leonardo Ferreira ${ }^{1 *}$, Elvi Cristina Rojas Fonseca ${ }^{1}$, Ana Luiza Rosa Lucas ${ }^{1}$, Adryelle Cristina Ferreira Silva ${ }^{1}$, Sarah Santiago Lenci ${ }^{1}$, Sarah Gazarra Ferreira Silva ${ }^{1}$, Fausto Jorge de Almeida Júnior $^{1}$, Flávia Hermano Rodrigues dos Santos ${ }^{1}$, Isabela de Paula Rezende ${ }^{1}$, Jussara da Cruz Jardim ${ }^{1}$, Roberta Garcia Leal Veiga Gonçalves ${ }^{1}$, Elisabete Aparecida Mantovani Rodrigues de Resende'.

\section{RESUMO}

Objetivos: Avaliar o impacto do aconselhamento multiprofissional sobre o conhecimento do doente acerca do Diabetes, observando a melhora dos parâmetros clínicos e laboratoriais e na aderência à hábitos e estilo de vida saudáveis. Métodos: Foi realizado um estudo descritivo e longitudinal, composto por pacientes diabéticos do tipo 1, de 4 a 18 anos e 2, de ambos os sexos com idade superior a 18 anos e que são atendidos rotineiramente no Ambulatório de Diabetes da Disciplina de Endocrinologia do HC-UFTM. A amostra do estudo foi composta por 51 pacientes Diabetes Mellitus tipo 2 (adultos) e 16 pacientes com Diabetes Mellitus tipo 1 (crianças e adolescentes). Foram realizados no mínimo 3 grupos com cada paciente. Os dados finais foram comparados com os dados iniciais (momento pré e pós). Resultados: A orientação preventiva ocasionou melhora nos dois grupos. As variáveis analisadas foram o autocuidado, controle e conhecimento da doença. Ocorreu melhora nos parâmetros clínicos (Glicemia Pós-Prandial, Colesterol Total e Frações, entre outros). Conclusão: $O$ estudo concluiu que a orientação preventiva multiprofissional em Diabetes traz resultados positivos para o paciente, tanto do tipo 1 quanto do tipo 2 .

Palavras-chaves: Diabetes, Aconselhamento multiprofissional, Orientação, Prevenção, Saúde.

\begin{abstract}
Objectives: Evaluate the impact of multiprofessional counseling on patients' knowledge about diabetes, observing the improvement of clinical and laboratory parameters and adherence to healthy habits and lifestyle. Methods: A descriptive and longitudinal study was carried consisting of type 1 diabetic patients, aged 4 to 18 years and 2, of both sexes over 18 years of age. routinely seen at the Diabetes Clinic of the Endocrinology Discipline of HC-UFTM. The study sample consisted of 51 Type 2 Diabetes Mellitus patients (adults) and 16 Type 1 Diabetes Mellitus patients (children and adolescents). At least 3 groups were performed with each patient. The final data were compared with the initial data (pre and post moment). Results: Preventive counseling improved both groups. The variables analyzed were self-care, control and knowledge of the disease. There was an improvement in clinical parameters (Postprandial Blood Glucose, Total Cholesterol and Fractions, among others. Conclusion: The study concluded that multiprofessional preventive guidance in Diabetes brings positive results for both type 1 and type 2 patients.
\end{abstract}

Keywords: Diabetes, Multidisciplinary counseling, Orientation, Prevention, Health.

${ }^{1}$ Universidade Federal do Triângulo Mineiro (UFTM), Uberaba - MG.

*E-mail: biologia.daniel89@gmail.com

SUBMETIDO EM: 11/2019

ACEITO EM: $12 / 2020$

PUBLICADO EM: 4/2020

REAS/EJCH | Vol.Sup.n.45 | e2381 | DOI: https://doi.org/10.25248/reas.e2381.2020 Página 1 de 11 


\section{RESUMEN}

Objetivos: Evaluar el impacto del asesoramiento multiprofesional en el conocimiento de los pacientes sobre diabetes, observando la mejora de los parámetros clínicos y de laboratorio y la adherencia a hábitos y estilos de vida saludables. Métodos: Se realizó un estudio descriptivo y longitudinal, compuesto por pacientes diabéticos tipo 1, de 4 a 18 años y 2, de ambos sexos mayores de 18 años. Visto habitualmente en la Clínica de Diabetes de la Disciplina de Endocrinología de HC-UFTM. La muestra del estudio consistió en 51 pacientes con diabetes mellitus tipo 2 (adultos) y 16 pacientes con diabetes mellitus tipo 1 (niños y adolescentes). Se realizaron al menos 3 grupos con cada paciente. Los datos finales se compararon con los datos iniciales (antes y después del momento). Resultados: El asesoramiento preventivo mejoró a ambos grupos. Las variables analizadas fueron autocuidado, control y conocimiento de la enfermedad. Parámetros clínicos mejorados (Glucosa en sangre posprandial, colesterol total y fracciones, entre otros. Conclusión: El estudio concluyó que la orientación preventiva multiprofesional en diabetes trae resultados positivos para los pacientes tipo 1 y tipo 2.

Palabras clave: Diabetes, Asesoramiento multidisciplinario, Orientación, Prevención, Salud.

\section{INTRODUÇÃO}

O Diabetes Mellitus (DM) consiste em um distúrbio metabólico caracterizado por hiperglicemia persistente, decorrente de deficiência na produção de insulina ou na sua ação, ou em ambos os mecanismos, ocasionando complicações em longo prazo. Atinge proporções epidêmicas, com estimativa de 415 milhões de portadores de DM mundialmente.

A hiperglicemia persistente está associada a complicações crônicas micro e macrovasculares, aumento de morbidade, redução da qualidade de vida e elevação da taxa de mortalidade. A classificação do DM tem sido baseada em sua etiologia. Os fatores causais dos principais tipos de DM - genéticos, biológicos e ambientais - ainda não são completamente conhecidos. Sabe-se que fatores como etilismo, tabagismo, sedentarismo e consumo inadequado de alimentos são fatores de risco relevantes para o desencadeamento da doença (SBD, 2017).

Denomina-se educação em diabetes o processo de desenvolvimento dessas habilidades, com a incorporação das ferramentas necessárias para atingir as metas estabelecidas em cada etapa do tratamento. Ela é, portanto, a principal ferramenta para a garantia do autocuidado que permitirá o autocontrole por parte do paciente (MULCAHY K, et al., 2003).

As orientações preventivas estão centradas às mudanças de hábitos do paciente em 3 vertentes: Estilo de vida, importância da realização de atividades físicas e o autocuidado. Obter hábitos é essencial no controle da glicemia, tanto para pacientes com Diabetes Mellitus do tipo 1 quanto do tipo 2. Entretanto, boa parte dos pacientes deixam de seguir as recomendações necessárias, como praticar atividades físicas por pelo menos 30 minutos, durante 5 dias da semana. Além disso, é muito importante seguir uma dieta balanceada, com quantidades adequadas de carboidratos, proteínas e gordura.

Cada paciente precisa de quantias próprias de cada grupo alimentar, portanto é preciso seguir orientações médicas e/ou nutricionais. Ainda é preciso controlar os fatores emocionais, visto que eles influenciam diretamente para complicações da doença (PERES HS, et al., 2016).

Assim, muitas vezes, torna-se necessário o autocuidado, ou seja, a condição do indivíduo de cuidar de si mesmo. Esse ensinamento é feito pelos profissionais da área da saúde. Grupos com paciente diabéticos, realizados por equipes multidisciplinares, ajudam pacientes que as vezes deixam de conhecer a gravidade das complicações do Diabetes, principalmente por não terem recebido ou assimilado informações. Geralmente, o tempo no consultório médico não é suficiente para serem tiradas todas as dúvidas do paciente, até pela alta quantidade de pacientes agendados nos hospitais pelo SUS (BRASIL, 2011).

Para tanto, o objetivo do trabalho foi a ocorrência da intervenção multiprofissional em saúde, para que esses dados sejam reduzidos, tanto os parâmetros clínicos e laboratoriais, quanto a capacidade do sujeito de conhecer melhor sua moléstia e aumentar o autocuidado. 


\section{MÉTODOS}

A amostra do estudo foi composta por 51 pacientes adultos (Diabetes Mellitus tipo 2) e 16 crianças e adolescentes (pacientes com Diabetes Mellitus tipo 1). Foram incluídos pacientes de ambos os sexos; Nível de alfabetização básico completo ou em andamento e além disso, pacientes que estejam participando da rotina de atendimentos do Ambulatório de Diabetes da Disciplina de Endocrinologia de um hospital do estado de Minas Gerais. Foram excluídos da pesquisa pacientes com Insuficiência Renal Crônica (IRC) e retinopatia grave.

As palestras foram elaboradas usando dois questionários, sendo o Diabetes Knowledge Questionnaire (DKN) validado por Torres HC, et al. (2005) que é um questionário autoaplicável e contém 15 itens de resposta de múltipla escolha acerca de diferentes aspectos relacionados ao conhecimento geral de diabetes mellitus. Apresentou cinco amplas categorias: fisiologia básica, incluindo a ação da insulina; hipoglicemia; grupos de alimentos e suas substituições; gerenciamento do diabetes na intercorrência de alguma outra doença, e princípios gerais dos cuidados da doença. Além disso, o questionário MICHAELS foi o instrumento de investigação das atividades de autocuidado do diabético. Foi aplicado quando ocorrer os primeiros eventos de orientação preventiva.

Os questionários serviram como roteiro de elaboração da palestra para a equipe multiprofissional. Foram identificados os déficits no conhecimento e atitudes frente ao autocuidado para DM e, a partir desse levantamento, as atividades educativas foram planejadas de maneira que pudessem incentivar os participantes a desenvolver atitudes corretas de autocuidado por meio da aquisição de novos conhecimentos sobre a doença. Os encontros aconteceram semanalmente, com duração em média de 40 minutos. Em todos eles, os pesquisadores e a equipe multiprofissional conduziam as atividades.

O período de intervenção durou nove meses, com 3 grupos semanais, exceto em semanas com feriados e pontos facultativos. Foram realizados 76 grupos no total. As estratégias utilizadas foram: Discussões que abordaram assuntos relativos à doença, tipos, causas, complicações e tratamento farmacológico e não farmacológico.

Contou-se com a participação ativa dos indivíduos, pois eles foram incentivados a falar seus conhecimentos e o modo como convivem com a doença; Oficinas educativas (dinâmicas de perguntas e respostas, colagens, dramatizações, atividades lúdicas) abordando assuntos relativos ao autocuidado para o manejo da doença; Apresentação de banner com a pirâmide alimentar e discussão sobre os aspectos nutricionais; exposição de fotos de complicações do DM; Distribuição de folders, panfletos e livretos educativos elaborados a partir dos déficits de conhecimentos identificados sobre a doença, suas complicações, o que fazer para controlar, sinais e sintomas de hipoglicemia e hiperglicemia e cuidados com os pés. Rodas de conversas para esclarecer as dúvidas dos participantes e manter um feedback do que foi assimilado; Atividades práticas para automonitorização glicêmica.

Para averiguar a evolução clínica dos pacientes, os pesquisadores convidaram os voluntários a retornarem em outras oportunidades, sempre quando frequentarem o ambulatório, para um novo grupo de aconselhamento. Para fazer parte da pesquisa, os pacientes compareceram no mínimo em 3 grupos de orientação.

Foi verificado, através de prontuário médico, dados como resultados de Exame de Glicemia em Jejum, Glicemia pós prandial, Hemoglobina Glicada, Triglicérides, Ferritina, Ácido Úrico, Transaminase Glutâmico Oxalacétiva, Transaminase Glutâmico Pirúvica, e gama Glutil - Transferase Colesterol Total e Frações, Pressão Arterial e peso corporal e esses dados foram armazenados no banco de informações do software Excel, comparando os exames iniciais e os exames feitos no final da pesquisa.

Os dados foram submetidos inicialmente à uma análise descritiva a partir de medidas de centralidade e dispersão. A comparação dos parâmetros de interesse antes e após o aconselhamento foi realizada a partir do teste t student pareado (quando normalidade dos dados satisfeita). Caso contrário, teste do Wilcoxon pareado. A normalidade dos dados foi verificada pelo teste de Shapiro-Wilk. O nível de significância para todos os testes foi de 5\%. Para verificar a relação do descontrole do Diabetes (pelas taxas de glicemia e hemoglobina glicada) com outros parâmetros clínicos laboratoriais, a análise se deu a partir inicialmente do cálculo do coeficiente de correlação linear de Pearson ou de Spearman, com ajuste de regressão linear ou 
não linear. O nível de significância adotado de $5 \%$. Para comparar a evolução dos grupos, utilizou - se o Teste Anova de Fator único, observando o valor de $\mathrm{p}$ e o nível de freqüência crítica. O nível de significância para todos os testes foi de $5 \%$. A análise estatística foi feita pelo programa SPSS versão 20,0 . O nível de significância para todos os testes foi de $5 \%$.

O estudo foi submetido ao Comitê de Ética em Pesquisa (CEP) da Universidade Federal do triângulo Mineiro (UFTM), obtendo aprovação, tendo como número de protocolo 2.385.154. Quando os pacientes compareciam ao ambulatório para consulta de rotina, eram abordados pelos pesquisadores que davam informações sobre a pesquisa e convidava-os a participar voluntariamente da mesma, com a apresentação verbal e por escrito do Termo de Consentimento Livre e Esclarecido (TCLE) para posteriormente preencherem e assinarem.

\section{RESULTADOS E DISCUSSÃO}

Inicialmente a amostra se constituiu de 71 pessoas com DM, porém, no final do estudo, foi excluída 1 participante por ser portadora de DM Gestacional e outras 3 deixaram de participar das palestras, não sendo localizadas pelos pesquisadores. Para caracterização das 67 pessoas com DM investigadas, descreveram-se aspectos referentes aos dados demográficos, dados clínicos e hábitos de vida.

Deste modo, participaram deste estudo, um total de 67 pessoas, sendo que a maior parte é do sexo feminino. Em relação à idade a faixa etária predominante situa-se entre os 6 e os 74 anos, fixando-se a média em 41,30 anos. A variação média do nosso estudo mostrou-se menor devida a característica de comparação entre os grupos de adultos $\mathrm{x}$ crianças, conforme Tabela 1.

Tabela 1 - Distribuição das variáveis sociodemográficas da população do estudo.

\begin{tabular}{ccc}
\hline Sexo & $\mathbf{n}$ & $\%$ \\
\hline Masculino & 30 & 44,7 \\
Feminino & 37 & 55,3 \\
\hline Faixa etária & & \\
\hline 4 a 11 & 8 & 12 \\
12 a 17 & 8 & 12 \\
18 a 74 & 51 & 76 \\
\hline Cor & & \\
\hline Branca & 45 & 67,2 \\
Preta & 13 & 19,4 \\
Parda & 9 & 13,4 \\
\hline Tipo DM & & 23,9 \\
\hline DM1 & 16 & 76,1 \\
\hline DM2 & 51 &
\end{tabular}

Fonte: Ferreira DL, et al., 2018.

Há estudos semelhantes que afirmam a prevalência de DM2 é maior em mulheres, e também, que elas procuram com mais frequência os serviços de saúde. As mulheres, em relação aos homens, se preocupam bem mais com a sua saúde e costumam se cuidar melhor, inclusive sendo as responsáveis pelo cuidado com a saúde de suas famílias. Portanto, elas estão mais atentas aos sinais e sintomas das doenças e, geralmente, procuram assistência com maior periodicidade (CAROLINO ID, et al., 2008).

As mulheres utilizam mais os serviços oferecidos pelas unidades primárias de atenção à saúde e procuram mais os médicos e os enfermeiros, sendo este um dos fatores que contribuem para maior expectativa de vida entre elas. E importante considerar esses dados para o planejamento da assistência à saúde. Uma vez que os homens retardam a sua procura pelos serviços de saúde, estão mais sujeitos a desenvolverem complicações pela falta de controle glicêmico e não adesão ao tratamento do DM. Um 
estudo realizado em unidade primária, com o objetivo de avaliar os efeitos da educação em grupos operativos de usuários com DM2 em relação aos conhecimentos e às práticas de autocuidado, mostrou média de idade de 63,2 anos (VIEIRA GCL, et al., 2011).

Com o avançar da idade, observa-se a presença de limitações na mobilidade física, na capacidade de autocuidado e de entendimento das informações recebidas sobre o DM. Desse modo, a idade é um fator importante a ser considerada no planejamento de um programa educativo, pois os jovens com diabetes aprendem desde cedo sobre a doença, já as pessoas idosas possuem níveis de dificuldade cognitiva diferenciados para a compreensão das informações e o cumprimento do plano terapêutico (BAQUEDANO IR, et al., 2010).

O estudo de Teston EF, et al. (2017) mostrou a importância do autocuidado, mencionando que o próprio sujeito é decisivo para a melhora de sua condição clínica. A melhora na prática do autocuidado, foi observado em ambos os grupos através dos critérios investigados (DM1 - monitorização da glicemia e DM2 - Aspectos negativos alimentação e cuidado com os pés).

Destaca-se no grupo DM1 a melhora na monitorização da glicemia seguida por boa parte da semana as orientações de um profissional de saúde. Por outro lado, o grupo DM1 apresentou um leve aumento de hábitos negativo de alimentação, como por exemplo consumo de doces. $(4,0-4,1)$. Já o grupo DM2 reduziu consideravelmente essa prática (4,0-2,9), mostrando melhor aproveitamento neste quesito. A SBD recomenda sempre manter hábitos saudáveis de alimentação, já que se trata de um fator de risco para desenvolver diabetes mellitus (SBD, 2017).

Os hábitos alimentares e a prática de atividades físicas exercem uma poderosa influência sobre o balanço energético, sendo considerados os principais fatores, passíveis de modificação, determinantes da obesidade (SARTORELLI RB, et al., 2003)

Um estudo com 225 pacientes diabéticos tipo 1 e 2, onde ele concluiu que os pacientes tipo 2 mostraram menor aderência às atividades físicas, por vários motivos (DUARTE CK, et al., 2015).

Nesse estudo, os pacientes tipo 2 também demonstraram menor frequência para realização de exercícios físicos. É importante o fim do sedentarismo para evitar doenças crônicas afirmando que o autocuidado se mostra fundamental até mesmo antes da doença "aparecer", pois há vários fatores que podem ser prevenidos e que contribuem para seu desenvolvimento como, por exemplo, o sedentarismo, estresse, obesidade e alimentação não saudável (SILVA JÁ, et al., 2018).

Ambos os grupos evoluíram na monitorização da glicemia, tendo como destaque o grupo DM1, onde teve significância estatística comprovada, que já apresentava boa prática desse item. A SBD (2017) traz a informação de que pacientes com DM2 fazem esquemas menos complexos de insulina e em sua maioria das vezes aferem menos a glicemia. Pacientes com DM2 que fazem uso de medicamentos orais parecem realizar menos aferições ainda. (4,1-4,3). Os resultados estão ilustrados na Tabela 2. 
Tabela 2 - Caracterização da população segundo as variáveis autocuidado em Diabetes (Michaels). Análise antes e depois das orientações preventivas multiprofissionais. Variáveis Autocuidado - antes e após grupos.

\begin{tabular}{|c|c|c|c|c|c|}
\hline \multirow[b]{2}{*}{ Diabetes mellitus 1} & \multicolumn{2}{|c|}{ Antes } & \multicolumn{2}{|c|}{ Após } & \multirow[b]{2}{*}{$\mathbf{P}$} \\
\hline & Média & D.P & Média & D.P & \\
\hline Aspectos Positivos Alimentação & 4,5 & 1,8 & 4,7 & 1,5 & $0,343^{1}$ \\
\hline Aspectos Negativos Alimentação & 4,0 & 1,4 & 4,1 & 1,6 & $0,861^{1}$ \\
\hline Aspectos Positivos Atividade Física & 4,4 & 1,8 & 4,3 & 1,8 & $0,446^{1}$ \\
\hline Monitorização Glicemia & 5,1 & 1,1 & 6,7 & 0,8 & $0,048^{1}$ \\
\hline Cuidado com os pés & 4,6 & 2,5 & 4,9 & 2,2 & $0,709^{1}$ \\
\hline Aspectos Positivos Medicação & 5,1 & 1,4 & 5,5 & 1,4 & $0,336^{1}$ \\
\hline Aspectos Positivos Gerais & 5,1 & 1,0 & 5,4 & 0,9 & $0,430^{1}$ \\
\hline \multicolumn{6}{|l|}{ Diabetes mellitus 2} \\
\hline Aspectos Positivos Alimentação & 4,3 & 2,1 & 4,6 & 1,3 & $0,727^{2}$ \\
\hline Aspectos Negativos Alimentação & 4,0 & 1,8 & 2,9 & 1,9 & $0,041^{1}$ \\
\hline Aspectos Positivos Atividade Física & 2,8 & 1,8 & 3,4 & 2,9 & $0,323^{2}$ \\
\hline Monitorização Glicemia & 4,1 & 2,9 & 4,3 & 2,7 & $0,087^{2}$ \\
\hline Cuidado com os pés & 3,9 & 2,5 & 5,8 & 2,1 & $0,039^{2}$ \\
\hline Aspectos Positivos Medicação & 5,4 & 1,8 & 5,4 & 1,7 & $0,655^{2}$ \\
\hline Aspectos Positivos Gerais & 4,3 & 1,5 & 4,6 & 1,3 & $0,111^{1}$ \\
\hline
\end{tabular}

Legenda: ${ }^{1}$ teste $t$ de Student para dados emparelhados. ${ }^{2}$ teste de Wilcoxon pareado. Fonte: Ferreira DL, et al., 2018. 
Os dois grupos pesquisados apresentaram melhora no conhecimento acerca do Diabetes. (DM1 = 60 $71,1)$ e $(D M 2=51-59,2)$, ilustrados nos gráficos 1 e 2 . O primeiro grupo obteve melhores resultados, provavelmente pelo fato dos pais e/ou acompanhantes orientarem as crianças e adolescentes a análise adequada das questões. Os pais não respondiam as questões para os filhos, mas auxiliavam os pesquisadores a deixarem claro a interpretação das perguntas, conforme mostra a Tabela 3.

Conforme o estudo foi desenvolvido, foi aplicado um questionário, do qual podemos evidenciar de forma resumida os seguintes assuntos discutidos: na diabete sem controle, o nível de açúcar no sangue é alto. Denomina-se hiperglicemia tal fato e pode ser causada pela falta de insulina. Já a segunda questão trouxe afirmações, sendo apenas uma correta. Algumas alegações menosprezam ou distorcem a importância do controle da diabetes ao focar apenas no livramento do coma ou o suposto benefício em ter pouca quantidade de açúcar na urina, evitando a hipoglicemia. Dentre elas, a única resposta correta seria que o controle mal feito da diabete pode resultar numa chance maior de complicações nas fases seguintes de vida do sujeito.

Na próxima questão, abordou-se a faixa de variação normal da glicose no sangue (uma pessoa que não é diabética), que em jejum, deve diversificar entre $70 \mathrm{mg} / \mathrm{dL}$ e $110 \mathrm{mg} / \mathrm{dL}$. Além dessa medida, similarmente foi identificado qual o conhecimento dos participantes sobre a unidade de peso, quilo, equivalente a mil gramas. Dessa forma, a noção sobre a composição dos alimentos também foi citada, sendo a manteiga constituída de gorduras do leite e o arroz de carboidratos, principalmente o arroz que não é integral (polido). Ainda no quesito alimentação, confirmou-se que agrião e alface são dois alimentos que os diabéticos podem comer o quanto desejarem. Duas substituições corretas foram expostas como exemplo para uma alimentação mais diversificada, sem deixar de ser saudável para o sujeito diabético. A primeira é substituir o pão francês por quatro biscoitos de água e sal, e a segunda é que um ovo é igual a uma porção de carne moída. Similarmente, para o café da manhã é permitido que se substitua o pão francês por quatro biscoitos de água e sal ou dois pães de queijo médios.

Sobre as complicações na diabetes, algumas são citadas como a presença de cetonas na urina, que é mau sinal, ou seja, o corpo está utilizando gordura para ter energia ao invés de usar glicose, ao ter como um dos possíveis motivos a insuficiência de insulina. Conhece-se muitos distúrbios e sintomas relacionados à diabetes como o pé diabético, a retinopatia, as lesões nos rins e que a hipoglicemia é causada por excesso de insulina. No entanto, uma complicação geralmente não associada à doença são as alterações nos pulmões.

Finalmente, como forma de empoderamento do diabético sobre sua condição e também do cuidador ou da pessoa próxima a ele, situações problema hipotéticas foram apresentadas e a melhor conduta foi a resposta das questões. A primeira se refere à pessoa que está tomando insulina, mas apresenta taxa alta de açúcar no sangue ou urina, assim como a presença de cetonas. O sujeito deve manter a mesma quantidade de insulina e dieta e fazer exame de sangue e urina. A segunda suposição é de que se uma pessoa diabética está tomando insulina e adoece ou não consegue comer a dieta receitada, deve-se usar hipoglicemiante oral para diabetes ao invés da insulina. Outra precaução versa sobre 0 início da hipoglicemia, momento em que é preciso que o sujeito coma ou beba algo doce imediatamente.

Tabela 3 - Caracterização da amostra segundo os níveis de erros e acertos sobre conhecimento do Diabetes (dkn questionnaire). Análise antes e depois das orientações preventivas multiprofissionais. Níveis de erros e acertos DKN

\begin{tabular}{|c|c|c|c|c|c|c|}
\hline Grupo & & Média & D.P & Média & D.P & $\mathbf{P}$ \\
\hline Acertos DM1 & 60 & & 19,33 & 71,1 & 17,4 & 0,0441 \\
\hline Acertos DM 2 & 51 & & 19,51 & 59,6 & 18,3 & $0,007^{1}$ \\
\hline FV & $S Q$ & gl & $M Q$ & $\mathrm{~F}$ & $\mathrm{P}$ & F crítico \\
\hline Entre grupos & 476,5633 & 2 & 236,282 & 16,18292 & $0,003299^{2}$ & 9,552094 \\
\hline Dentro dos grupos & 107,125 & 3 & 35,70833 & & & \\
\hline Total & 583,6883 & 5 & & & & \\
\hline
\end{tabular}

Legenda: ${ }^{1}$ teste t de Student para dados emparelhados. ${ }^{2}$ ANOVA - F. Único.

Fonte: Ferreira DL, et al., 2018. 
Gráfico 1 - Percentual (\%) de acertos - grupo DM1.

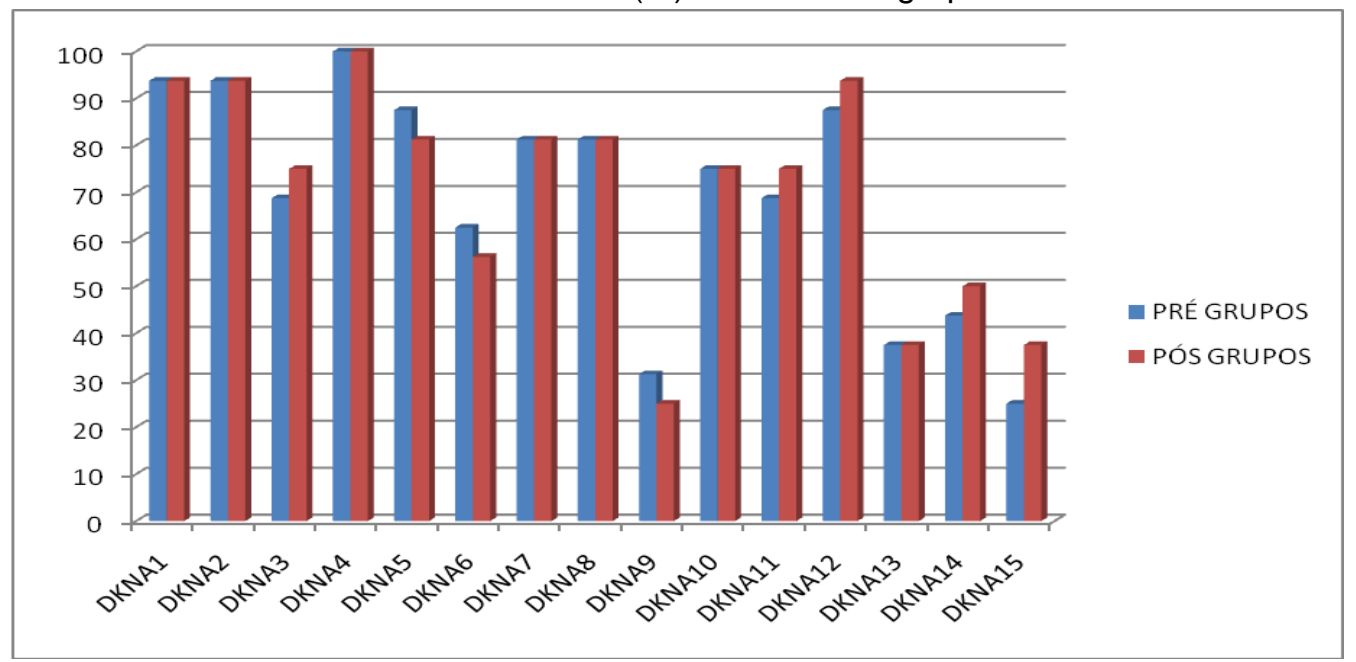

Fonte: Ferreira DL, et al., 2018.

Gráfico 2 - Percentual (\%) de acertos - grupo DM 2.

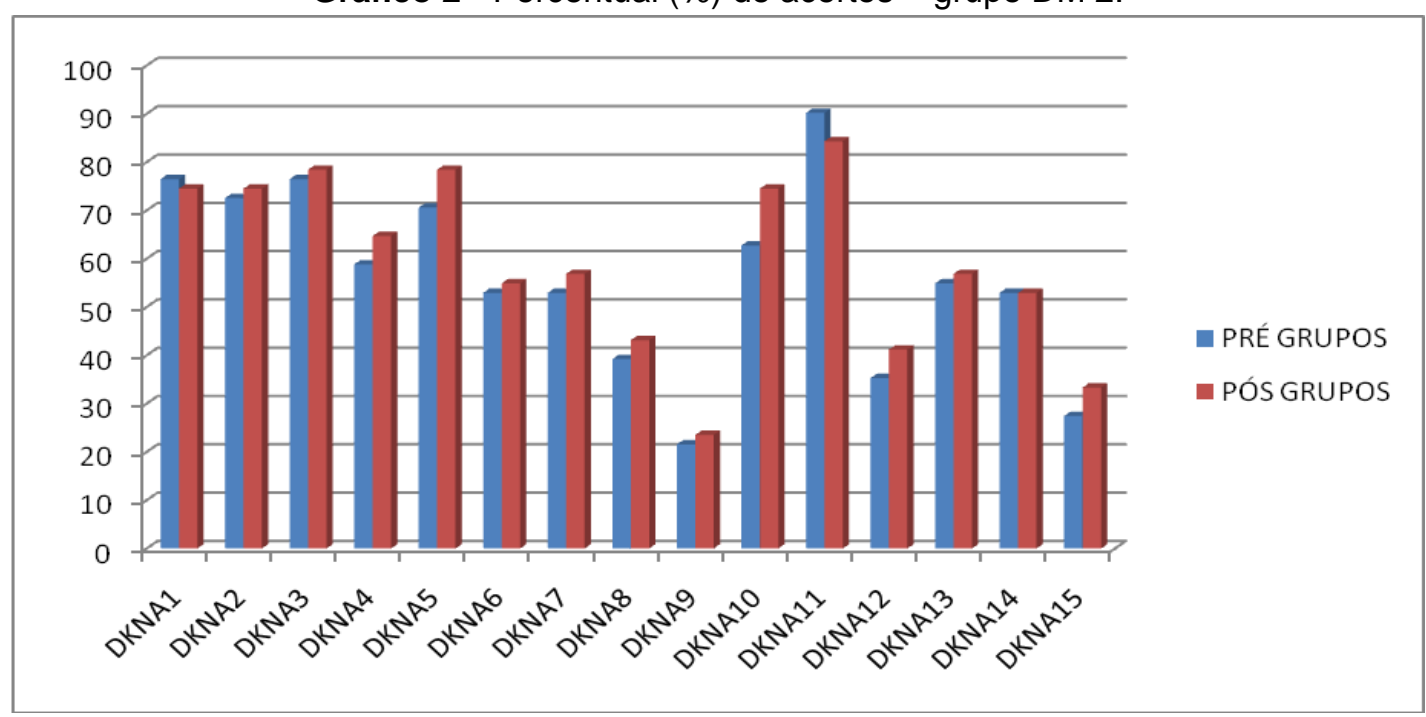

Fonte: Ferreira DL, et al., 2018.

Estudos sugerem que o tempo da doença e a escolaridade refletem diretamente no conhecimento do paciente perante a doença. As atividades educativas realizadas no estudo de Oliveira (2011), foram organizadas através de atividades lúdicas e pedagógicas direcionadas ao tipo de público específico e, quando necessária, constituído por um conjunto de metodologias voltadas para a fisiopatologia da doença. A educação em saúde é fundamental para elevar o conhecimento do paciente e melhorar sua adesão ao tratamento, inclusive em comunidades com diferentes características socioeconômicas e culturais (OLIVEIRA KCS, et al., 2011).

Quanto à glicemia capilar pós-prandial, Lopes, (2018) realizou um estudo com características semelhantes a esse, a maioria entrevistados estavam com glicemia alterada e poucos indivíduos com valores normais. Obteve-se uma diminuição considerável da glicemia pós-prandial $(258,9-214,2)$ e discreto aumento da glicemia de jejum no grupo DM1 (195,2-205,2).

Quanto ao colesterol total e frações, houve queda de LDL significante no grupo DM2 (DM2 $=107,5-$ $86,6)$ com redução de triglicérides. O peso corporal praticamente se manteve, ou diminuiu, em ambos os grupos.

As pressões arteriais sistólicas e diastólicas mantiveram-se dentro dos parâmetros normais, com ambos os grupos apresentando ligeira queda da pressão sistólica e ligeiro aumento na diastólica. Os outros 
parâmetros clínicos (TGO, TGP, Gama GT e Ácido Úrico) melhoraram ou se mantiveram próximos aos índices anteriores, evitando a piora no controle do Diabetes como mostra a Tabela 4.

Tabela 4 - Comparação das médias do exame clínico antes e depois da realização das orientações preventivas multiprofissionais. Parâmetros Clínicos Laboratoriais

\begin{tabular}{cccccc}
\hline & \multicolumn{2}{c}{ Antes } & \multicolumn{2}{c}{ Após } \\
& Média & D. padrão & Média & D. padrão & P \\
\hline Diabetes mellitus $\mathbf{1}$ & & & & & \\
\hline Glicemia de Jejum & 195,2 & 66,7 & 205,2 & 71,8 & $0,487^{1}$ \\
Glicemia pós & 258,9 & 64,3 & 214,2 & 77,5 & $0,033^{1}$ \\
prandial & 10,3 & 3,9 & 9,6 & 2,2 & $0,776^{2}$ \\
Hba1c & 153,9 & 18,7 & 170,1 & 15,9 & $0,569^{1}$ \\
Colesterol total & 49,2 & 5,6 & 66,6 & 9,7 & $0,347^{1}$ \\
Hdl & 94,3 & 17,3 & 89,5 & 15,3 & $0,573^{1}$ \\
Ldl & 77,8 & 19,5 & 59,6 & 18,6 & $0,047^{1}$ \\
Triglicérides & 43,8 & 18,1 & 47,6 & 16,7 & $0,128^{1}$ \\
Peso & 124,0 & 8,9 & 123,5 & 7,7 & $0,564^{2}$ \\
Pressão sistólica & 73,0 & 4,2 & 81,0 & 7,2 & $0,414^{2}$ \\
Pressão diastólica & & & & \\
\hline Diabetes mellitus 2 & & & 176,3 & 74,3 & $0,658^{2}$ \\
\hline Glicemia de Jejum & 187,4 & 65,2 & 234,5 & 135,8 & $0,572^{2}$ \\
Glicemia pós & 226,4 & 110,9 & 8,6 & 2,4 & $0,793^{1}$ \\
prandial & 8,8 & 2,2 & 163,3 & 37,7 & $0,107^{1}$ \\
Hba1c & 176,2 & 45,8 & 50,1 & 15,6 & $0,422^{1}$ \\
Colesterol total & 48,1 & 20,7 & 76,6 & 36,4 & $0,041^{2}$ \\
Hdl & 107,5 & 37,8 & 153,3 & 97,2 & $0,882^{1}$ \\
Ldl & 154,8 & 67,6 & 74,3 & 16 & $0,363^{1}$ \\
Triglicérides & 74,1 & 16,3 & 128,75 & 20,6 & $0,550^{2}$ \\
Peso & 131,5 & 21,8 & 81,4 & 11,8 & $0,267^{1}$ \\
Pressão sistólica & 80,3 & 11,4 & &
\end{tabular}

Legenda: ${ }^{1}$ teste $t$ de Student para dados emparelhados. ${ }^{2}$ teste de Wilcoxon pareado.

Fonte: Ferreira DL, et al., 2018.

A educação em saúde é fundamental para pessoas acometidas por doenças crônicas, considerando o autocuidado, mudança dos hábitos e melhoria da qualidade de vida. A ideia central do grupo operativo é uma abordagem para o usuário, com o objetivo na promoção de sua autonomia, além de oferecer espaço e diálogo para troca suas experiências, condições de vida, saúde e doença (LEMOS CA, et al., 2016).

Para os estudos em Patologia do DM, contribui para o reforço da importância do tratamento não medicamentoso, orientação preventiva e autocuidado. Como limitação do estudo, percebeu-se a falta de um espaço físico adequado para a atividade de realização da "Sala de Espera", aproveitando o tempo dos pacientes até serem atendidos em suas consultas. Tanto as crianças, quanto os adultos responderam positivamente após as intervenções. Novos estudos são necessários para reforçar ainda mais essa hipótese.

\section{CONCLUSÃO}

O estudo concluiu que a orientação preventiva multiprofissional em Diabetes traz resultados positivos para o paciente diabético, tanto do tipo 1 quanto do tipo 2. Contribui para a melhora dos parâmetros clínicos, devido ao fato de incentivar os hábitos corretos. Traz o aumento do conhecimento do diabético, inclusive melhorando a prática do autocuidado. Para os estudos em Patologia do DM, contribui para o reforço da importância do tratamento não medicamentoso, orientação preventiva e autocuidado. Como 
limitação do estudo, percebeu-se a falta de um espaço físico adequado para a atividade de realização da "Sala de Espera", aproveitando o tempo dos pacientes até serem atendidos em suas consultas. Tanto as crianças, quanto os adultos responderam positivamente após as intervenções. Novos estudos são necessários para reforçar ainda mais essa hipótese.

\section{REFERÊNCIAS}

1. AMERICAN DIABETES ASSOCIATION. Standards of medical care in diabetes. Diabetes Care. 2017; 40(Suppl 1): S1-131.

2. BAQUEDANO IR, et al. Autocuidado de personas con Diabetes Mellitus atendidas en un servicio de urgencia en México. Revista Latino-Americana de Enfermagem, 2010; 18(6): 1195-1202.

3. BARBOZA SM. Influência da qualidade de vida no controle glicêmico de pacientes com Diabetes mellitus tipo 2. 2012. Dissertação (Mestrado em Ciências) - Faculdade de Medicina. Universidade de São Paulo, Ribeirão Preto. 2012; p. 66-7.

4. BOSCO A, et al. Retinopatia diabética. Arquivos Brasileiros de Endocrinologia e Metabologia. 2005; 49(2): 217-27.

5. CARVALHO FPB, et al . Prevalência de doença arterial coronariana em pacientes diabéticos. Revista de Enfermagem UFPE online. 2016; 10(2): 750-5.

6. CAROLINO ID, et al. Fatores de risco em pacientes com Diabetes Mellitus tipo 2. Revista Latino-Americana de Enfermagem. 2008; 16(2): 1-7.

7. COSTA JR, et al. Desfechos clínicos tardios de pacientes diabéticos tratados com stents farmacológicos eluidores de sirolimus ou everolimus: uma análise do registro DESIRE. Revista Brasileira de Cardiologia Invasiva. 2011; 23(1): 17-21.

8. CORRÊA FH, et al. Avaliação da secreção e resistência insulínica em indivíduos com diferentes graus de tolerância à glicose - do metabolismo normal ao diabetes mellitus. Arquivos Brasileiros de Endocrinologia e Metabologia. 2007; 51(9): 1498-505.

9. D'AGOSTINO RB, et al. General cardiovascular risk profile for use in primarycare: the Framingham Heart Study. Circulation. 2008; 117(6): 743-53.

10. DAVIDSON J, BERGENSTAL, RM. Glucose pattern management teaches glycemiarelated problem-solving skills in a diabetes self-management education program. Diabetes Spectrum. 2013; 26: 91-7.

11. DUARTE CK, et al. Nível de atividade física e exercício físico em pacientes com diabetes mellitus. Revista da Associação Médica Brasileira. 2012; 58(2): 215-21.

12. EVANGELISTA VC, et al. Equipe multiprofissional de terapia intensiva: humanização e fragmentação do processo de trabalho. Revista Brasileira de Enfermagem. 2016; 69(6): 1099-107.

13. FRONTERA WR, et al. Exercício físico e reabilitação. Porto Alegre: Artmed Editora, 2001; 420p.

14. GOMES MB. Glitazonas e síndrome metabólica: mecanismos de ação, fisiopatologia e indicações terapêuticas. Arquivos Brasileiros de Endocrinologia e Metabologia. 2006; 50(2): 271-80.

15. GROSS JL, et al. Diabetes melito: diagnóstico, classificação e avaliação do controle glicêmico. Arquivo Brasileiro de Endocrinologia e Metabologia. 2002; 46: 16-26.

16. KITABACHI A, et al. Hyperglycemic crises in adult patients with diabetes. Diabetes Care. 2009; 32(7): 1335-43.

17. KLAFKE A, et al. Mortalidade por complicações agudas do diabetes melito no Brasil, 2006-2010. Epidemiologia e Serviços de Saúde. 2014; 23(3): 455-62.

18. INTERNATIONAL DIABETES FEDERATION. IDF Atlas. 7th ed. Brussels, Belgium: International Diabetes Federation, 2015; 142p.

19. ISER B, et al. Prevalência de diabetes autorreferido no Brasil: resultados da Pesquisa Nacional de Saúde 2013. Epidemiologia e Serviços de Saúde. 2015; 24(2): 305-14.

20. SANTOMAURO JÚNIOR AC, et al. Metformina e AMPK: um antigo fármaco e uma nova enzima no contexto da síndrome metabólica. Arquivos Brasileiros de Endocrinologia e Metabologia. 2008; 52(1): 120-5.

21. PORTH CM. Fisiopatologia. 8. ed. Rio de Janeiro: Guanabara Koogan, 2010; 2v.

22. POVOA T, et al. Treinamento aerobio e resistido, qualidade de vida e capacidade funcional de hipertensas. Revista Brasileira de Medicina do Esporte. 2014; 20(1): 36-41.

23. LANDIM CA. A competência de pessoas com diabetes mellitus para o autocuidado em um programa educativo multiprofissional. Dissertação (Mestrado em Enfermagem) - Escola de Enfermagem de Ribeirão Preto. Universidade de São Paulo, Ribeirão Preto, 2009; 152p.

24. LERARIO AC, et al. Algorithm for the treatment of type 2 diabetes: a position statement of Brazilian Diabetes Society. Diabetology \& Metabolic Syndrome. 2010; 2:35.

25. LYRA RA, et al. Diabetes melito: classificação e diagnóstico. In: VILAR L, editor. Endocrinologia clínica. 6. ed. Rio de Janeiro: Guanabara Koogan, 2016; p. 641-56.

26. LOPES LV. Programa educativo para o autocuidado de pessoas com Diabetes mellitus: subsídio para o cuidado clínico de enfermagem. 2015. Dissertação (Mestrado em Cuidados Clínicos em Enfermagem e Saúde) - Centro de Ciências da Saúde. Universidade Estadual do Ceará, Fortaleza, 2015; 114p.

27. LOTTENBERG AS, et al. Manual de Diabetes Mellitus. Liga de Controle de Diabetes Mellitus do Hospital das Clinicas da Faculdade de Medicina-USP. São Paulo: Atheneu, 2010; 326p.

28. MICHELS MJ, et al. Questionário de Atividades de Autocuidado com o Diabetes: tradução, adaptação e avaliação das propriedades psicométricas. Arquivos Brasileiros de Endocrinologia e Metabologia. 2010; 54(7): 644-51. 
29. BRASIL. Ministério da Saúde. Secretaria de Atenção à Saúde. Departamento de Atenção Básica. Diabetes mellitus. Brasília, DF: Ministério da Saúde, 2013; 160p. (Cadernos da Atenção Básica; 36).

30. BRASIL. Ministério da Saúde. Prevenção clínica de doenças cardiovasculares, cerebrovasculares e renais. Brasília, DF: Ministério da Saúde, 2006; 58p. (Cadernos de Atenção Básica; 14).

31. MULCAHY K, et al. Diabetes self-management education core outcomes measures. Diabetes Educator. 2003; 29(5): 768-70.

32. OLIVEIRA K, et al. Conhecimento e atitudes de usuários com diabetes mellitus em um Serviço de Atenção Básica à Saúde. Revista da Escola de Enfermagem da USP. 2011; 45(4): 862-8.

33. PERES HS, et al. Lifestyle of patients with diabetes mellitus type 1: a systematic review. Ciência \& Saúde Coletiva. 2016; 21(4): 1197-206.

34. PREIS S, et al. Trends in all-cause and cardiovascular disease mortality among women and men with and without diabetes mellitus in the Framingham Heart Study, 1950 to 2005. 2009; 119(13): 1728-35.

35. QUERES JFN. Evaluation of knowledge and attitudes and their relation to Diabetes mellitus control parameters. Journal of Health Connections. 2018; 2(1): 21-9.

36. RADIGONDA R, et al. Avaliação do acompanhamento de pacientes adultos com hipertensão arterial e ou diabetes melito pela Estratégia Saúde da Família e identificação de fatores associados. Epidemiologia e Serviços de Saúde. 2016; 25(1): 115-26.

37. ROCHA KS. Diabetes mellitus: o conhecimento dos pacientes acerca do autocuidado. Revista interdisciplinar. 2015; 8(1): 98-106.

38. BARREIRO-RIBEIRO $F$, et al. Hypertriglyceridemic waist phenotype indicates insulin resistance in adolescents according to the Clamp Technique in the BRAMS study. Child Obesity. 2016; 12(6): 446-54.

39. CAMPOS FCC, FARIA HP. Planejamento e avaliação das ações em saúde. 2. ed. Belo Horizonte: Nescon/UFMG, 2010; 118p.

40. SOCIEDADE BRASILEIRA DE DIABETES. Diretrizes da Sociedade Brasileira de Diabetes: 2017-2018. São Paulo: AC Farmacêutica, 2014; 382p. 